\title{
Mecanismos de influencia educativa en un Preuniversitario Popular de Santiago de Chile: control del aprendizaje y equidad en la educación superior*
}

\author{
Mechanisms of educational influence in a Popular Pre-University of Santiago: \\ control of learning and equity in higher education \\ Mecanismos de influência educacional num Cursinho Pre-vestibular \\ de Santiago do Chile: control da aprendizagem e equidade no ensino superior
}

\author{
Paulina Castro Torres, ${ }^{a}$ Romina Aranda Cáceres $^{b}$ \\ aPrograma de Investigación en Equidad en Educación Superior (PIEES), \\ Departamento de Psicología, Universidad de Chile \\ Telf.: 229787716. Correo electrónico: paucastr@u.uchile.cl \\ bPrograma de Investigación en Equidad en Educación Superior (PIEES), \\ Departamento de Psicología, Universidad de Chile \\ Telf.: 229787791. Correo electrónico: romina.arandac@gmail.com
}

\begin{abstract}
RESUMEN
Desde la psicología instruccional y una mirada socioconstructivista se discute la labor de un preuniversitario popular en el desarrollo de equidad en el acceso a la educación superior chilena. Se describe como mecanismo de influencia educativa el traspaso del control y la responsabilidad en el proceso de aprendizaje desde el profesor al estudiante, en dos secuencias didácticas de temas evaluados en la Prueba de Selección Universitaria: matemáticas y lenguaje. La muestra fue intencionada y constituida por un grupo de egresados de liceos Técnico Profesionales del preuniversitario popular durante el año 2011. Se produjo la información mediante observaciones y registros de audio de clases que fueron analizados según segmentos de interactividad y actuaciones. Se observaron similitudes y diferencias entre las secuencias didácticas estudiadas, así como indicadores de este mecanismo que resulta crucial para una mayor equidad educativa.
\end{abstract}

Palabras clave: influencia educativa, preuniversitarios populares, equidad.

ABSTRACT
This paper discusses the role of a popular preparatory school to Universities' admission exams in developing equity access to Chilean Higher Education from the instructional psychology and the social constructivism perspective. The transference of control and the responsibility of the learning process from the teacher to the student are described as an educational influence mechanism in two didactic sequences of evaluated themes in the 'University Selection Exam': Mathematics and Language. The sample was directed and constituted by a group of graduates from Technical High Schools that attended a popular preparatory school to Universities' admission exams during 2011. The data was collected through observations and classes audio records, which were analyzed according to segments of interactivity and performances. Similarities and differences between teaching sequences studied were observed, as well as indicators of this mechanism, which are crucial for greater educational equity.

Key words: educational influence, popular preparatory school to Universities' admission exams, equity.

\footnotetext{
Esta investigación fue financiada por el Fondo Semilla de Investigación 2011 del Departamento de Psicología de la Facultad
} de Ciencias Sociales de la Universidad de Chile. 


\section{INTRODUCCIÓN}

En Chile existen graves obstáculos en el acceso a la educación superior universitaria para los y las estudiantes pertenecientes a los tres primeros quintiles de ingreso económico (Aguilar, 2010; Brunner, 2008; Cancino y Donoso, 2007; Catrileo, 2010; Cinda, 2010, 2011; Comité Técnico Asesor CRUCH, 2010; Espinoza y González, 2011; Latorre, González y Espinoza, 2007; Manzi, 2006), razón por la cual desde hace un tiempo la temática de equidad en Educación Superior (ES) ha cobrado relevancia política y sin duda académica.

Hablar de equidad desde el punto de vista de la justicia social, implica adentrarse en las teorías de justicia distributiva, cuya perspectiva de la educación complejiza la noción de igualdad de oportunidades a un nivel formal, en tanto "el hecho de considerar a todos por igual puede resultar en que se dé un trato desigual a aquellos que se encuentran en una posición desfavorable" (Bolívar 2005: 43). Así, la equidad es pensada en términos de una distribución equitativa de los bienes y las oportunidades educativas, con el fin de que las desigualdades no afecten el aprendizaje y el rendimiento de los estudiantes. Para ello, los medios compensatorios deben apuntar y encontrarse al servicio de aquellos más desfavorecidos (Bolívar, 2005). Para Neves de Azevedo (2013) el ejercicio de la equidad y de la igualdad sustantiva supone la supresión de los sistemas discriminatorios de distribución existentes en sociedades capitalistas. De esta forma, el objetivo de las acciones afirmativas que se encuentran bajo el alero de la equidad, solo puede ser plenamente alcanzado en una fase post-revolucionaria.

De un modo operativo, el concepto de equidad en ES puede ser analizado desde el modelo propuesto por Latorre et al. (2007), que considera las siguientes dimensiones: a) Acceso, b) Permanencia: mantención en el sistema, c) Logros: expresados en las calificaciones, y d) Resultados: representados por las posibilidades de trabajo, ingresos y ejercicio de poder político una vez egresado.

Respecto del acceso, cabe señalar que en el sistema educativo chileno este aspecto se relacionaría con desigualdades socioeconómicas debido a la presencia de una alta segregación por clases sociales. Lo anterior se produce principalmente por dos razones: la baja cobertura curricular que se alcanza en los liceos y la imposibilidad de que el segmento más pobre de estudiantes pueda asistir y costear un preuniversitario (OCDE \& Banco Mundial, 2009). De hecho, los preuniversitarios han sido calificados como un factor más de segregación y discriminación educativa (Eyzaguirre y Le Foulon, 2002; Williamson y Rodríguez, 2010), ya que afectan el proceso de transición entre la educación media y superior, en la medida que funcionan como "operadores de cursos remediales mercantiles y extra sistema que distorsionan las posibilidades de ingreso de aquellos jóvenes que no disponen de los recursos para financiar estas opciones" (González, 2011: 25). En este contexto, los preuniversitarios populares, organizados e impartidos por educadores que muchas veces son estudiantes universitarios de distintas carreras y universidades, surgen como una alternativa extra sistema que permite acceder a un servicio de este tipo a postulantes que por razones económicas no lo pueden hacer.

En Santiago de Chile, actualmente una parte de los preuniversitarios populares están organizados en la Red de Preuniversitarios de la Universidad de Chile (PREURED, 2016) ${ }^{1}$, sin embargo, para el año 2012, siete de estos preuniversitarios estaban organizados en

Mayor información en http://preured.cl/ 
la Región Metropolitana en la Red de Preuniversitarios, ofreciendo durante el mismo año una matrícula de más de 3200 cupos. Cabe señalar que estas organizaciones se mantienen gracias al trabajo de los educadores y la colaboración de organizaciones no gubernamentales, escuelas, liceos o universidades que prestan el espacio físico para que se desarrollen las clases, que en algunos casos no tienen costo para el alumno o bien un mínimo cobro que no supera los 100 USD anuales.

Respecto del preuniversitario en el cual se desarrolló este estudio, para el año 2010 el Preuniversitario de Estudiantes de la Facultad de Medicina de la Universidad de Chile (PREUMED) $)^{2}$ contaba con un cupo de 500 matrículas, las cuales estaban completas casi en su totalidad. Los cursos se organizaban en grupos de aproximadamente 80 personas cada uno. Por otra parte, los resultados obtenidos por PREUMED durante el mismo año, alcanzaron un promedio de 530 puntos en la Prueba de Selección Universitaria chilena (PSU) de Matemáticas, y sobre los 550 puntos en la PSU Lenguaje y Ciencias.

En la mayoría de los casos estos educadores carecen de formación pedagógica formal, al mismo tiempo que presentan la necesidad constante de mejorar sus estrategias de enseñanza para favorecer los aprendizajes y por ende los resultados de los estudiantes en la PSU -en especial de aquellos estudiantes que inician el preuniversitario con un nivel más bajo de rendimiento en los ensayos PSU de matemáticas y lenguaje, debido principalmente a su egreso de liceos técnico-profesionales, que en Chile comparativamente cuentan con menos horas de formación en ambas áreas del currículum nacional de enseñanza media.

Como en todos los preuniversitarios populares, en tanto instituciones educativas, en PREUMED existe intencionalidad de enseñanza y aprendizaje de aquella selección de contenidos definidos y elaborados culturalmente, y que en este caso corresponden al conjunto de contenidos y habilidades que evalúa la PSU. El presente estudio adopta una perspectiva de la enseñanza y del aprendizaje coherente con el enfoque sociocultural del desarrollo de las funciones psicológicas superiores (Wertsch, 1988), especificada por Vygotsky (1988) y retomada más tarde por algunas perspectivas socio constructivistas de la enseñanza y el aprendizaje (Coll, 1995). Conceptos cruciales en esta línea de investigación psicoeducativa son la Zona de Desarrollo Próximo (Vigostky, 1988), Andamiaje (Wood, Brunner y Ross, 1976), y participación guiada (Rogoff, 1993).

Desde el enfoque sociocultural, los procesos psicológicos como el aprendizaje, son entendidos como el resultado de la mutua interacción entre el individuo y la cultura, en donde la construcción de significados y más específicamente de aquellos que se atribuyen a los objetos, palabras y acciones de los otros (Wertsch, 1988) resulta fundamental. Siguiendo esta idea, el proceso por medio del cual se llega a conocer el significado de las cosas, es dado a partir de las relaciones que se establecen con otros actores sociales, así como también, de la intervención intencionada de los adultos en el aprendizaje de los niños o aprendices. Esto ocurre a través de una mediación de tipo predominantemente semiótica que se da muy claramente en los procesos de instrucción, en donde se evidencian interacciones, especialmente lingüísticas, entre profesor, alumno y pares. En este proceso, el significado de los signos será resultado de una negociación entre los participantes (Martínez, 1999; Wertsch, 1988).

Considerando lo anterior, el aprendizaje puede definirse como un "proceso activo de construcción de significados por parte de los alumnos que se realiza a partir de la puesta en

Mayores antecedentes pueden ser revisados en: http://www.preumeduchile.cl/?page_id=2 
relación del conocimiento previo ya existente en la propia estructura cognitiva con el nuevo material de aprendizaje" (Onrubia, 1993: 84). Ahora bien, en dicho proceso, la labor de quien enseña ocupa un rol primordial en tanto responsable de orientar y guiar (Coll, Onrubia \& Mauri, 2008; Onrubia, 1993; Rogoff, 1993) la actividad constructiva desarrollada por los estudiantes en relación a contenidos previamente definidos y elaborados culturalmente (Onrubia, 1993). Dicho de otro modo, se considera que el aprendizaje de contenidos y habilidades de los estudiantes es producto de la ayuda brindada por sus enseñantes a través de diversos mecanismos de tipo interpsicológico, denominados mecanismos de influencia educativa (Coll et al., 1992; Coll, 1995; Coll et al., 2008; Onrubia, 1993), los cuales operan en el marco de la interactividad o de la articulación del triángulo didáctico: contenido, docente y estudiantes (Coll y Solé, 2002). Desde el punto de vista de la interactividad, los dos mecanismos de influencia educativa más relevantes son: el traspaso del control y la responsabilidad en el proceso de aprendizaje, desde los/as docentes a los estudiantes, y la construcción progresiva de niveles de intersubjetividad que aseguren que ambos actores alcancen representaciones comunes en el desarrollo de la actividad conjunta en el aula (Onrubia, 1993). Para efectos del presente trabajo, se describirá el primero de ellos.

La aproximación a estos dos grandes procesos de influencia educativa en el aula se realiza a través de la identificación de las características de la actividad conjunta, y el análisis de la actividad discursiva y no discursiva de profesores y alumnos en el contexto de secuencias didácticas (Coll et al., 2008; Colomina, Mayordomo y Onrubia, 2001). Al respecto, variados son los trabajos que se fundamentan en una concepción socioconstructivista de la enseñanza y el aprendizaje, con foco en los mecanismos de influencia educativa. Tatiana Goldrine (2007), por ejemplo, buscó describir la práctica docente a través del estudio de la interactividad profesor-estudiantes; Rosemberg (2000) analiza dichos mecanismos durante los primeros años de escolaridad; y Buitrago y Gutiérrez (2009) analizan los mecanismos de influencia educativa en dos escenarios: uno presencial y uno semi presencial con uso de TICS. Hallazgos interesantes, en algunos casos compartidos por escenarios educativos diferentes, muestran el aumento de segmentos de interactividad asociados a la práctica, ejecución o aplicación del conocimiento por parte de los y las aprendices (Maiz, Zarandona y Arrieta, 1996; Onrubia, 1993). Asimismo, se da cuenta de la importancia de la materia de estudio y el o la docente en las características de los segmentos de interactividad que se observan (Coll et al., 2008; Zarandona, Arrieta, Maiz, 1997).

Considerando la evidencia teórica disponible, primero que todo, es posible comprender a los preuniversitarios populares como lugares estratégicos en la construcción de equidad, ya que influencian el proceso de transición de la enseñanza media a la universitaria en un espacio en donde ni el mercado despliega lucro, ni el estado desarrolla políticas. Esto, debido a su capacidad de promover el desarrollo de la dimensión de acceso del modelo de equidad en la educación superior (Latorre et al., 2007), al ser espacios que desarrollan mecanismos de influencia educativa $($ Coll, 1995) con una clara intencionalidad educativa referida al currículum evaluado (Glatthorn, 2006) en la PSU de Chile. Segundo, los preuniversitarios populares se producen a través de la autogestión, considerada el máximo nivel de participación en una organización educativa (Heredero y Bris, 2005), constituyendo espacios que han optado por desarrollar objetivos políticos a través de la educación, o como lo denomina Giroux (1983), espacios de resistencia que son poco (re) conocidos en el sistema académico y de los cuales en Chile se ha producido poca literatura especializada. 
De acurdo a lo anterior, el objetivo general del estudio fue describir los mecanismos de influencia educativa, referidos al traspaso del control y la responsabilidad del aprendizaje desde el profesor hacia el estudiante, y la construcción progresiva de significados compartidos entre ambos, en dos secuencias didácticas de PSU, Matemáticas y Lenguaje, en un grupo compuesto mayoritariamente por egresados de educación técnico profesional del Preuniversitario Popular PREUMED durante el año 2011. Para efectos de este artículo, se presentarán y discutirán los resultados del primero de sus objetivos específicos, es decir, la identificación del mecanismo de influencia educativa referido al traspaso del control sobre el aprendizaje desde el profesor hacia los estudiantes en dos secuencias didácticas de PSU Matemáticas y Lenguaje.

\section{MÉTODO}

El presente corresponde a un estudio de tipo exploratorio y descriptivo, que utiliza el modelo de enfoque dominante, es decir, adopta de forma preponderante la perspectiva del enfoque cualitativo, pero integrando también cierto componente cuantitativo en su desarrollo (Hernández, Fernández y Baptista, 2006).

El muestreo fue de tipo intencionado, las clases específicas y la selección de los participantes (grupo a observar) se definió en función de la demanda presentada por los educadores en torno a recibir información para la mejora de sus prácticas pedagógicas, especialmente en el curso constituido por estudiantes egresados de liceos técnico profesionales. En PSU matemáticas se trabajó en la clase de "Ecuaciones", registrada durante el 29 de mayo entre las 18:00 y 20:00 horas. En lenguaje se trabajó con la clase de "Plan de Redacción", observada los días 30 de mayo y 3 de junio de 18:00 a 20:00 hrs. En ambas materias se efectuaron observaciones previas de otras clases con el fin de aproximarse de manera progresiva a la metodología de trabajo, y además para que los participantes se acostumbraran a la situación de observación.

Se negoció con el conjunto de profesores cercanos a la gestión del preuniversitario popular las características que tendría el trabajo, que por lo demás, contó con la participación voluntaria de profesores y estudiantes.

Para la producción de información se utilizó en gran medida el procedimiento propuesto por Coll et al. (2008; Coll, 1995, 2002; Coll y Solé, 2002; Coll et al., 1995; Colomina et al., 2001), sin embargo, se desarrollaron algunas adaptaciones en función de las posibilidades existentes en el espacio de terreno. Se llevó a cabo observación no participante y registro de campo de las actuaciones de profesores y estudiantes durante el desarrollo de las secuencias didácticas de aspectos no verbales. Junto con esto se registró en audio la clase para recoger los aspectos verbales de la interactividad entre los actores. Finalmente y previo al análisis se integraron ambos registros. Siguiendo la propuesta de Coll et al. (2008) y Colomina et al. (2001), se distinguieron dos niveles de análisis de la información, cuyos resultados se apoyan y enriquecen mutuamente con el fin de elaborar una interpretación global e integrada de los datos. No obstante lo anterior, para fines de este artículo, como se indicó, se describirá tan solo el primero de ellos.

El objetivo del primer nivel de análisis consiste en identificar formas de organización de la actividad conjunta, es decir, patrones de actuación entre profesores y estudiantes a lo largo de la Secuencia Didáctica, que corresponde a la unidad básica de análisis, dentro de la 
cual es posible identificar segmentos de interactividad (SI) que constituyen "un fragmento de actividad conjunta que presenta una determinada estructura de participación y que mantiene una unidad temática" (Coll et al., 2008: 45). Las configuraciones de segmentos de interactividad (CSI) que articulan los segmentos, corresponden a una unidad de análisis de segundo orden en este nivel.

El procedimiento de análisis consideró, en primer lugar, la identificación y descripción de segmentos de interactividad y de configuraciones de segmentos de interactividad en el caso de que existieran, siendo básico para ello la identificación de patrones de actuación típicos de estudiantes y profesores en el aula, es decir, la identificación de las expresiones verbales y no verbales manifestadas por los participantes y la vinculación de dichas actuaciones entre ambos actores.

Luego, la interpretación de las funciones instruccionales de cada SI desde la influencia educativa, el análisis de evolución de los segmentos de interactividad y de las configuraciones de estos, así como la construcción de mapas de interactividad de las secuencias didácticas. Además, se realizó el análisis de evolución de patrones de actuación de profesores y estudiantes para cada tipo de SD. Por último, se interpretó de manera global este primer nivel de análisis referido a la identificación y caracterización de formas de organización de la actividad conjunta.

En la identificación de los indicadores anteriormente descritos se aplicó como procedimiento el análisis, primero por separado y luego en conjunto entre ambas investigadoras, sirviendo además como una forma de triangular la información, ya que proporcionó miradas diferentes. A continuación se presentarán los segmentos de interactividad, junto a sus patrones de actuación característicos, así como el mapa de interactividad de cada secuencia didáctica, entregando una perspectiva de la evolución en el tiempo de dichos segmentos.

\section{PRINCIPALES RESULTADOS}

Los Segmentos de Interactividad, como grandes clasificaciones de la actividad conjunta, son producto de numerosos y minuciosos procesos de ida y vuelta hacia los registros. Por tanto, corresponden a unidades construidas a posteriori y no predeterminadas por el diseño de la investigación. Es así como luego de sucesivas revisiones en base a los criterios expuestos, se ha llegado a proponer SI característicos de cada momento de la $\mathrm{SD}$, tanto en matemáticas como en lenguaje. A continuación, se indican los patrones de actuación más característicos, y en el caso de los segmentos más importantes se presenta la estructura interna y ejemplos representativos de estos.

En el caso de la SD de matemáticas, se categorizaron cuatro segmentos de interactividad: Introducción (SII), Explicación participativa (SIEP), Aclaración de dudas (SIAC) y Ejecución individual (SIEI). En lenguaje se categorizaron cinco segmentos de interactividad: Introducción (SII); Evaluación (SIEV), Explicación (SIE), Análisis de ejemplos (SIAE) y Ejercitación conjunta (SIEC).

El segmento de interactividad de introducción (SII) fue identificado en ambas secuencias didácticas, pero en diferentes momentos de esta. En matemáticas se ubica siempre al comienzo de cada una de las tres partes (ver Mapa de Interactividad, Gráfico 1) que componen la clase, mientras que en lenguaje esta no se presenta regularidad. 
Como aspecto común, se observa que en ambas materias este segmento es iniciado por el o la docente, y su objetivo correspondería al encuentro entre los diferentes actores, la explicitación del objetivo de la clase y la entrega de información administrativa. La configuración de actuaciones y las actuaciones que predominan son los actos de cortesía (AC) e indicaciones normativas (IN) por parte de la docente, y murmullos (MUR) por parte de los estudiantes.

En ambas SD se producen segmentos de interactividad dedicados a la explicación de las materias. En el caso de matemáticas, ha sido posible identificar el SI de explicación participativa (SIEP), que surge posteriormente a la introducción (ver Mapa de Interactividad, Gráfico 1), siendo iniciado por la educadora y cuyo objetivo consiste en que los estudiantes comprendan la temática que ha sido indicada en la introducción. Es participativa ya que la profesora promueve el intercambio entre los estudiantes, quienes también explican y responden consultas en la clase. En el caso de lenguaje, se categorizó el SI de explicación (SIEX) que, al igual que el anterior, pretende la comprensión del tema en torno al cual se organiza la secuencia didáctica, mas sin promover la intervención de los estudiantes.

En ambas asignaturas, las actuaciones y configuraciones de actuaciones que predominan en el anterior tipo de segmento son explicaciones por parte del o la docente y seguimiento de los alumnos (EXP/SEG) ${ }^{3}$, pregunta del o la docente, respuesta de el o los estudiante y feedback del o la profesora $(\mathrm{P}-\mathrm{R}-\mathrm{F})^{4}$, y pregunta del o la docente y respuesta del o los estudiantes (PREG-RESP). En el caso de lenguaje, además se apreciaron dos configuraciones de actuaciones relevantes: pregunta del estudiante, respuesta del profesor e indicación de comprensión del estudiante (P-R-IC); y pregunta por parte del profesor, ausencia de respuesta por parte de los alumnos y explicación por parte del profesor (P-AR-E).

Este tipo de segmento, a diferencia del anterior, es clave en el trabajo de los docentes de ambas asignaturas. Esto se aprecia, en el caso de matemáticas, en el tiempo de duración total (82 minutos), así como también en su distribución, observándose en los tres momentos de la clase. En lenguaje, por el contrario, este patrón predomina en momentos iniciales de la $\mathrm{SD}$, en donde el profesor mantiene más o menos constante su actuación de explicar a los estudiantes lo que ellos no son capaces de responder. A medida que avanza la actividad conjunta, el docente de lenguaje cambia de estrategia: al no obtener respuesta de los alumnos, generalmente vuelve a insistir en la pregunta, hasta que obtiene una respuesta y logra cerrar el patrón (P-AR-E).

La tabla que se presenta a continuación (Tabla $\left.\mathrm{N}^{\circ} 1\right)$ muestra ejemplos de los patrones de actuación EXP/SEG, P-R-F, y P-AR-E de ambas materias en segmentos dedicados a la explicación (SIEP, SIEX). En las columnas de la izquierda se muestra la configuración o patrón de actuación y se identifica la materia y segmento, y en las columnas de la derecha se presentan los ejemplos del respectivo patrón:

El signo / indica actuaciones que ocurren al mismo tiempo. Siempre se codifica primero la actuación del profesor y a continuación la actuación del estudiante, para luego nuevamente codificar la actuación del profesor y así sucesivamente, hasta que finaliza el patrón.

$4 \quad$ El signo - indica actuaciones consecutivas en el tiempo. 
MECANISMOS DE INFLUENCIA EDUCATIVA EN UN PREUNIVERSITARIO POPULAR DE SANTIAGO DE CHILE: CONTROL DEL APRENDIZAJE Y EQUIDAD EN LA EDUCACIÓN SUPERIOR

Tabla 1. Patrones de Actuación SIEP de matemáticas y SIEX de lenguaje

\begin{tabular}{|c|c|c|c|c|}
\hline & \multicolumn{2}{|c|}{$\begin{array}{c}\text { Patrón de Actuación EXP/SEG } \\
\text { matemáticas (SIEP) }\end{array}$} & \multirow{2}{*}{$\begin{array}{c}\text { Ejemplo de Actuación } \\
\text { Profesora }\end{array}$} & \multirow{2}{*}{$\begin{array}{c}\text { Ejemplo de Actuación } \\
\text { Estudiante }\end{array}$} \\
\hline & Profesora & Estudiante & & \\
\hline \multirow[t]{3}{*}{ a) } & Explicación & Seguimiento & $\begin{array}{l}\text { Pa: Si sumo aquí los exponentes, } \\
\text { me da 'x' cubo. Y eso es lo mis- } \\
\text { mo que si yo tengo < raíz cuadra- } \\
\text { da de 'x' por raíz cuadrada de 'x' } \\
\text { [dice la profesora] }\end{array}$ & [Miran a la profesora] \\
\hline & \multicolumn{2}{|c|}{$\begin{array}{l}\text { Patrón de Actuación P-R-F } \\
\text { matemáticas (SIEP) }\end{array}$} & Ejemplo de Actuación & Ejemplo de Actuación \\
\hline & Profesora & Estudiante & Profesora & Estudiante \\
\hline \multirow[t]{4}{*}{ b) } & $\begin{array}{l}\text { Pregunta } \\
\text { (interrogación) }\end{array}$ & \multirow[t]{2}{*}{ respuesta } & $\begin{array}{l}\text { Pa: ¿qué pasa con esto? [señala } \\
\text { una parte de la ecuación] }\end{array}$ & \multirow[t]{2}{*}{$\begin{array}{l}\text { E: Se simplifica [un grupo } \\
\text { de estudiantes ha } \\
\text { respondido] } \\
\text { E: Se simplifica. }\end{array}$} \\
\hline & Feedback & & Pa.: Se simplifica, ¿no cierto? & \\
\hline & \multicolumn{2}{|c|}{$\begin{array}{c}\text { Patrón de Actuación P-AR-E } \\
\text { lenguaje (SIEX) }\end{array}$} & Ejemplo de Actuación & Ejemplo de Actuación \\
\hline & Profesora & Estudiante & Profesora & Estudiante \\
\hline \multirow[t]{2}{*}{ c) } & Pregunta & \multirow[t]{2}{*}{$\begin{array}{l}\text { Ausencia de } \\
\text { respuesta }\end{array}$} & Po: ¿Se entiende? & \multirow[t]{2}{*}{$\begin{array}{l}\text { E: [No hay respuesta de } \\
\text { los estudiantes] }(0.1)\end{array}$} \\
\hline & Explicación & & $\begin{array}{l}\text { Po: Ya... es casi lo mismo, pri- } \\
\text { mero voy a poner idea más gene- } \\
\text { ral, definición y después el ori- } \\
\text { gen... [Vuelve a mirar diapositiva } \\
10] \text { y en las biografías por lo ge- } \\
\text { neral se hace una breve reseña de } \\
\text { qué es lo que estamos hablando } \\
(0.2) \text { después voy a empezar a en- } \\
\text { tregar la información con otro } \\
\text { criterio, por lo general el cronoló- } \\
\text { gico (0.2) Ehh... Otro criterio de } \\
\text { ordenación es el estructural [cam- } \\
\text { bia de diapositiva a la } 11 \text { ], que es } \\
\text { como típico de los ensayos... } \\
\end{array}$ & \\
\hline
\end{tabular}

Por otra parte, en ambas asignaturas se aprecian segmentos que permiten la explicitación de consultas de los estudiantes, aunque con formas de organización de la actividad conjunta diferentes según la materia. En matemáticas, surgió el segmento de interactividad de aclaración de dudas (SIAD) con posterioridad al SI de Explicación (ver Mapa de Interactividad matemáticas, Gráfico $\mathrm{N}^{\circ} 1$ ), siendo iniciado por un estudiante que pregunta o por la profesora que invita a preguntar. Su objetivo es dar respuesta a las interrogantes de los estudiantes, derivadas de explicaciones previas. Predominan actuaciones de pregunta 
por parte del estudiante y de respuesta por parte de la docente (SEG/PREG-RESP), a veces seguida de indicación de comprensión por parte del estudiante (P-R-IC).

En lenguaje, en cambio, el anterior tipo de segmento se denominó segmento de interactividad de análisis de ejemplos (SIAE), ubicándose con posterioridad al segmento de explicación y en la primera sesión de la SD (ver Mapa de Interactividad lenguaje, Gráfico $\mathrm{N}^{\circ} 2$ ). Este segmento constituye en lenguaje una instancia cuyo objetivo es revisar ejemplos de ejercicios resueltos de manera conjunta. El profesor es quien va explicando la lógica de resolución de los ejercicios, sin embargo, los alumnos tienen la posibilidad de intervenir. Los patrones de actuación dominantes son: explicación-seguimiento (EXP/ SEG), pregunta, respuesta, feedback (P-R-F), y preguntas realizadas por los estudiantes y seguidas por el profesor, y que son respondidas por este último (SEG/PREG-RESP).

A continuación se describen ejemplos de los patrones de actuación predominantes en este grupo de segmentos: SEG/PREG-RESP, P-R-IC y EXP/SEG.

Tabla 2. Patrones de Actuación SIAD matemáticas y SIAE lenguaje

\begin{tabular}{|c|c|c|c|c|}
\hline & \multicolumn{2}{|c|}{$\begin{array}{c}\text { Patrón de Actuación SEG/ } \\
\text { PREG-RESP } \\
\text { matemáticas (SIAD) }\end{array}$} & Ejemplo de Actuación & Ejemplo de Actuación \\
\hline & Profesora & Estudiante & Profesora & Estudiante \\
\hline \multirow[t]{4}{*}{ a) } & seguimiento & \multirow[t]{2}{*}{ pregunta } & (escucha y mira al estudiante) & \multirow{2}{*}{$\begin{array}{l}\text { Ao: Ah, perdón. ¿Cuál es } \\
\text { el índice? }\end{array}$} \\
\hline & respuesta & & $\begin{array}{l}\text { pa.: Esto es el índice... [lo señala } \\
\text { en la pizarra] }\end{array}$ & \\
\hline & \multicolumn{2}{|c|}{$\begin{array}{c}\text { Patrón de Actuación P-R-IC } \\
\text { matemáticas (SIAD) }\end{array}$} & Ejemplo de Actuación & Ejemplo de Actuación \\
\hline & Profesora & Estudiante & Profesora & Estudiante \\
\hline \multirow[t]{4}{*}{ b) } & \multirow[t]{2}{*}{ respuesta } & pregunta & \multirow{2}{*}{$\begin{array}{l}\text { Pa: ...y esto es el exponente [lo } \\
\text { señala en la pizarra] }\end{array}$} & Ao: $Y$ el exponente... \\
\hline & & $\begin{array}{l}\text { Indicación de } \\
\text { comprensión }\end{array}$ & & $\begin{array}{l}\text { Ao: Ah, ya. En el índice } \\
\text { cuando... }\end{array}$ \\
\hline & \multicolumn{2}{|c|}{$\begin{array}{c}\text { Patrón de Actuación EXP/SEG } \\
\text { lenguaje (SIAE) }\end{array}$} & Ejemplo de Actuación & Ejemplo de Actuación \\
\hline & Profesora & Estudiante & Profesora & Estudiante \\
\hline c) & Explicación & Seguimiento & $\begin{array}{l}\text { P1: Ya. Nominación. Este es de la } \\
\text { vida real [Lee diapositiva]: Los ni- } \\
\text { ños del Preu. Este año entraron al } \\
\text { Preu muchos alumnos nuevos. En- } \\
\text { tre ellos están Miguel, Jorge y Ca- } \\
\text { mila. Miguel es muy simpático y } \\
\text { le gusta mucho conversar en clase }\end{array}$ & E: [Prestan atención] \\
\hline
\end{tabular}


En ambas materias se aprecian segmentos de interactividad dedicados al desarrollo, por parte de los estudiantes, de ejercicios de una guía de trabajo. En matemáticas, se categorizó el segmento de interactividad de ejecución individual (SIEI), el que se ubica entre SI de explicación participativa y aclaración de dudas individuales (ver Mapa de Interactividad matemáticas, Gráfico $\mathrm{N}^{\circ} 1$ ), y es iniciado por la docente. Los patrones de actuación que caracterizan este segmento corresponden a la solicitud de ejecución por parte de la profesora, y ejecución por parte del estudiante (SOLEJE-EJE), Pregunta-Respuesta (P$\mathrm{R})$, Pregunta-Respuesta-Feedback (P-R-F), y también interacciones en las que el alumno realiza un ejercicio en la pizarra en tanto va respondiendo a las preguntas de la profesora y el resto del curso murmura (P-R/MUR).

En el caso de lenguaje, este tipo de segmento aparece posterior al segmento de explicación y fue denominado segmento de interactividad de ejercitación conjunta (SIEC) (ver Mapa de Interactividad lenguaje, Gráfico $\mathrm{N}^{\circ} 2$ ), se caracterizó por el desarrollo de ejercicios de manera conjunta entre el profesor y los estudiantes en base a una guía de trabajo. En este segmento los alumnos son los encargados de ir configurando la respuesta correcta y el profesor se convierte solo en una guía para la concreción de dicho objetivo. Este momento es iniciado por el docente y en él predominan los siguientes patrones de actuación: Explicación-Seguimiento (EXP-SEG), Pregunta-Respuesta-Feedback (P-R-F), Pregunta-Respuesta (PREG-RESP), y preguntas realizadas por el docente que no logran obtener una respuesta por parte de los estudiantes (P-AR). Es preciso señalar que en este segmento, ante las ausencias de respuesta, el profesor tiende a insistir en la pregunta a través reformulaciones, con el objeto de que los estudiantes finalmente sean capaces de ejecutarlas, y de este modo, cerrar el patrón.

A continuación se presenta una tabla (Tabla $\mathrm{N}^{\circ} 3$ ) que muestra ejemplos de la articulación de actuaciones SOLEJE-EJE, P-R/MUR, P-AR y P-R-F de la profesora o profesor y los estudiantes. En las dos primeras columnas de la derecha se describe lo que dicen/hacen el o la profesora y estudiantes así como también la asignatura correspondiente, y en las otras dos columnas, el ejemplo de la actuación:

Tabla 3. Patrones de Actuación SIEI matemáticas y SIAC lenguaje

\begin{tabular}{|c|c|c|c|c|}
\hline & \multicolumn{2}{|c|}{$\begin{array}{l}\text { Patrón de Actuación } \\
\text { SOLEJE-EJE } \\
\text { matemáticas (SIEI) }\end{array}$} & \multirow{2}{*}{$\begin{array}{c}\text { Ejemplo de Actuación } \\
\text { Profesora }\end{array}$} & \multirow{2}{*}{$\begin{array}{c}\text { Ejemplo de Actuación } \\
\text { Estudiante }\end{array}$} \\
\hline & Profesora & Estudiante & & \\
\hline \multirow[t]{2}{*}{ a) } & $\begin{array}{l}\text { Solicitud de } \\
\text { ejecución }\end{array}$ & & \multirow[t]{2}{*}{$\begin{array}{l}\text { pa.: ¿Sí? Ya. Ahora que alguien } \\
\text { salga a la pizarra a hacer el nue- } \\
\text { ve. Que sea voluntario, por favor. } \\
\text { [La profe ahora está adelante de } \\
\text { la sala mira a los alumnos] }\end{array}$} & \\
\hline & & Ejecución & & $\begin{array}{l}\text { Ao: Yo. [se dirige a la pi- } \\
\text { zarra] [sube un poco la } \\
\text { murmuración] }\end{array}$ \\
\hline
\end{tabular}


Estudios Pedagógicos XLII, N² 2: 51-68, 2016 MECANISMOS DE INFLUENCIA EDUCATIVA EN UN PREUNIVERSITARIO POPULAR DE SANTIAGO DE CHILE: CONTROL DEL APRENDIZAJE Y EQUIDAD EN LA EDUCACIÓN SUPERIOR

\begin{tabular}{|c|c|c|c|c|}
\hline & \multicolumn{2}{|c|}{$\begin{array}{c}\text { Patrón de Actuación P-R/MUR } \\
\text { matemáticas (SIEI) }\end{array}$} & \multirow{2}{*}{$\begin{array}{c}\text { Ejemplo de Actuación } \\
\text { Profesora }\end{array}$} & \multirow{2}{*}{$\begin{array}{c}\text { Ejemplo de Actuación } \\
\text { Estudiante }\end{array}$} \\
\hline & Profesora & Estudiante & & \\
\hline \multirow[t]{4}{*}{ b) } & \multirow[t]{2}{*}{ pregunta } & Murmullos & \multirow[t]{2}{*}{$\begin{array}{l}\text { Entonces, ¿qué voy a hacer? } \\
\text { [pregunta la profesora a la estu- } \\
\text { diante que está en la pizarra]. }\end{array}$} & $\begin{array}{l}\text { [los alumnos murmuran } \\
\text { más }]\end{array}$ \\
\hline & & $\begin{array}{l}\text { Respuesta/ } \\
\text { murmullos }\end{array}$ & & Aa: ¿Pasarla sumando? \\
\hline & \multicolumn{2}{|c|}{$\begin{array}{l}\text { Patrón de Actuación P-R-F } \\
\text { lenguaje (SIEC) }\end{array}$} & Ejemplo de Actuación & Ejemplo de Actuación \\
\hline & Profesora & Estudiante & Profesora & Estudiante \\
\hline \multirow[t]{3}{*}{ b) } & Pregunta & & $\begin{array}{l}\text { P1: ¿Por qué? [P1 no alcanza a } \\
\text { escuchar explicación de E1] A } \\
\text { ver, que alguien levante la mano } \\
\text { y explique... }\end{array}$ & \\
\hline & & Respuesta & & $\begin{array}{l}\text { E1: [levanta la mano] } \\
\text { Porque primero... es } \\
\text { un... a ver, es un... un } \\
\text { enunciado que es más } \\
\text { plural que el resto, el otro } \\
\text { es más específico. Enton- } \\
\text { ces hay que abocarse más } \\
\text { que nada primero a lo plu- } \\
\text { ral y luego a lo específico. }\end{array}$ \\
\hline & Feedback & & $\begin{array}{l}\text { P1: Es más global [hace énfasis } \\
\text { en la pronunciación de la pala- } \\
\text { bra], más global. Más que plural. } \\
\text { Plural tiene que ver con cantidad. } \\
\text { Lo más global... }\end{array}$ & \\
\hline
\end{tabular}

Además de los segmentos ya mencionados, de modo específico en la SD de lenguaje se distingue el segmento de interactividad de Evaluación (SIEV), referido al tiempo dedicado para la evaluación del conocimiento de los estudiantes, mediante la realización de un minitest individual. Este momento es iniciado por el docente y en él predominan los siguientes patrones de actuación: solicitud de ejecución por parte del profesor y ejecución por parte de los estudiantes (SOLEJE-EJE), así como también indicaciones normativas (IN) y murmullos (MUR) unidas a actuaciones como ejecución (EJE) o seguimiento (SEG) respectivamente. A continuación se aprecian algunos ejemplos de dichos patrones en la Tabla 4: 
MECANISMOS DE INFLUENCIA EDUCATIVA EN UN PREUNIVERSITARIO POPULAR DE SANTIAGO DE CHILE: CONTROL DEL APRENDIZAJE Y EQUIDAD EN LA EDUCACIÓN SUPERIOR

Tabla 4. Patrones de Actuación SIEV lenguaje

\begin{tabular}{|c|c|c|c|c|}
\hline & \multicolumn{2}{|c|}{$\begin{array}{l}\text { Patrón de Actuación } \\
\text { IN/EJE/MUR }\end{array}$} & \multirow{2}{*}{$\frac{\text { Ejemplo de Actuación }}{\text { Profesor }}$} & \multirow{2}{*}{$\begin{array}{c}\text { Ejemplo de Actuación } \\
\text { Estudiante }\end{array}$} \\
\hline & Profesor & Estudiante & & \\
\hline a) & $\begin{array}{l}\text { Indicación } \\
\text { Normativa }\end{array}$ & $\begin{array}{l}\text { Ejecución // } \\
\text { Murmullos }\end{array}$ & $\begin{array}{l}\text { P1: El minitest como todos, es } \\
\text { acerca de lo que vimos la clase } \\
\text { pasada }(0.30)\end{array}$ & $\begin{array}{l}\text { E: [Murmullos] // [realiza- } \\
\text { ción de minitest] }\end{array}$ \\
\hline \multirow[t]{4}{*}{ d) } & \multirow[t]{2}{*}{ Seguimiento } & & \multirow[t]{2}{*}{$\begin{array}{l}\text { P1: [Observa quehacer de los } \\
\text { estudiantes] }\end{array}$} & \\
\hline & & \multirow[t]{2}{*}{$\begin{array}{l}\text { Acto } \\
\text { disruptivo }\end{array}$} & & \multirow{2}{*}{$\begin{array}{l}\text { E: [Llegan dos alumnos atra- } \\
\text { sados] // [los demás alumnos } \\
\text { se encuentran realizando mi- } \\
\text { nitest] }\end{array}$} \\
\hline & \multirow{2}{*}{$\begin{array}{l}\text { Solicitud de } \\
\text { ejecución }\end{array}$} & & \multirow[t]{2}{*}{ P: [P1 entrega minitest] } & \\
\hline & & Ejecución & & $\begin{array}{l}\text { E: [los alumnos toman asien- } \\
\text { to en sector B y comienzan a } \\
\text { realizar prueba] }\end{array}$ \\
\hline
\end{tabular}

Finalmente, para apreciar la evolución en el tiempo de los segmentos de interactividad anteriormente descritos, se presenta el Mapa de Interactividad de matemáticas (Gráfico $\mathrm{N}^{\circ} 1$ ) y de lenguaje (Gráfico $\mathrm{N}^{\circ}$ 2). Cada barra muestra los segmentos de interactividad en el tiempo, ordenados de abajo hacia arriba, de acuerdo al momento que ocupan dentro de cada secuencia didáctica.

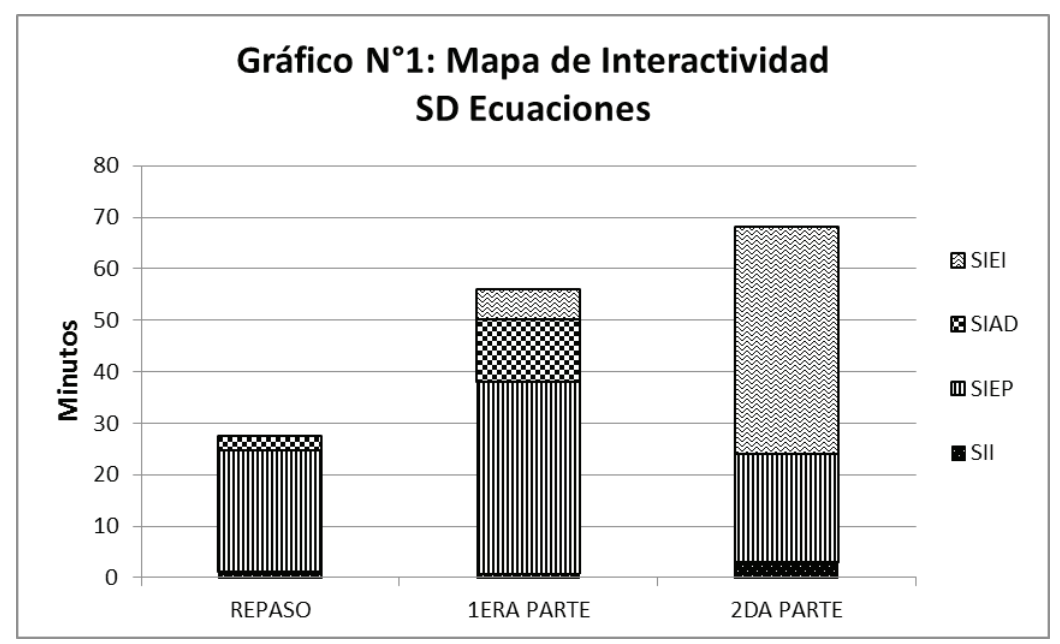




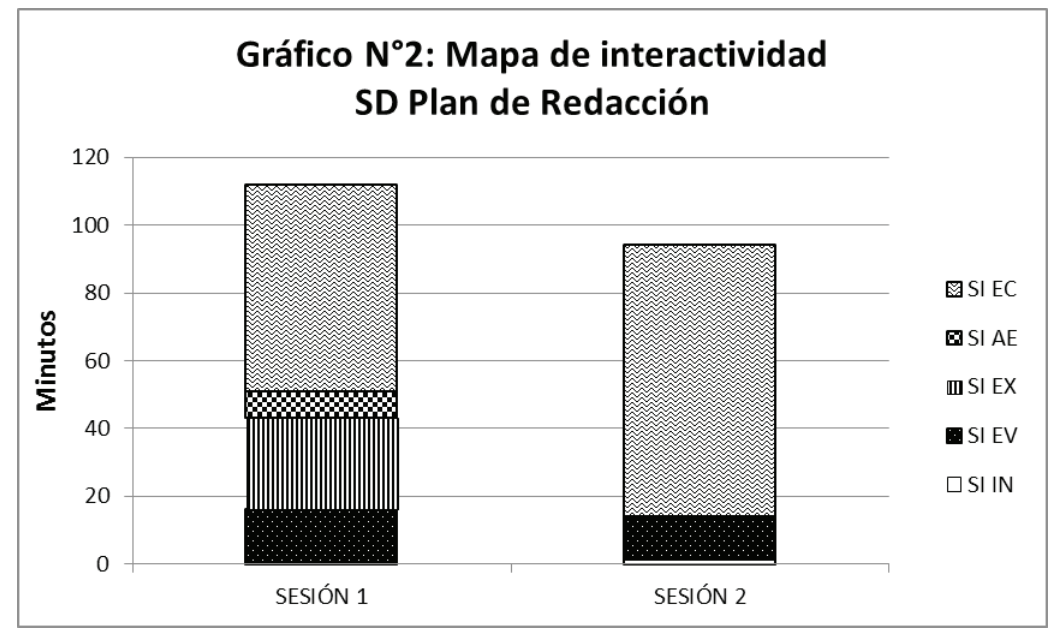

En el caso de matemáticas, se observa cierta configuración de segmentos de interactividad, ya que en los tres momentos de la clase aparece el segmento de introducción seguido de explicación participativa (SIIN+SIEP). En lenguaje no se distinguieron configuraciones de segmento de interactividad.

\section{DISCUSIÓN Y CONCLUSIONES}

Respecto al objetivo planteado, se apreciarían similitudes y diferencias entre las SD de matemáticas y lenguaje en relación a los mecanismos de influencia educativa vinculados con el traspaso del control y la responsabilidad en el aprendizaje, desde el docente hacia los estudiantes.

En primer lugar, es posible observar en ambas materias segmentos destinados a introducir el tema (SIIN), y segmentos dedicados a que el profesor explique dicho tema (SIEP, SIEX). En el caso de matemáticas la profesora agrega la variante de ir promoviendo, a lo largo de la secuencia didáctica, mayor participación de los estudiantes a través de la explicación entre pares. Asimismo, se evidencian patrones de actuación similares tanto en los segmentos de introducción (SIIN) como en los segmentos de explicación (SIEP, SIEX), caracterizados por la exposición del docente y el seguimiento por parte de los estudiantes (EXP-SEG). Ambos profesores incorporan de manera importante la pregunta y el feedback, y en el caso de los estudiantes predomina tanto la respuesta como la ausencia de esta (P-R-F, P-R, P-AR-F). Es posible ver este tipo de segmentos en las partes iniciales de la secuencia didáctica, disminuyendo su duración en el tiempo en la última parte de la clase de matemáticas, y desapareciendo para la segunda sesión en lenguaje, pudiendo ser este un indicador del grado de adecuación de la ayuda que entregan los educadores a sus estudiantes, según el nivel de competencia percibido por los primeros en la apropiación de los instrumentos, conceptos, habilidades o conocimientos (Brunner, 1991; Guilar, 2009; Wood et al., 1976) de sus alumnos. En los demás segmentos, las diferencias entre ambas 
secuencias didácticas se encuentran bien marcadas en lo que respecta a los mecanismos utilizados por los docentes para, desde los segmentos de explicación, andamiar (Brunner, 1991; Guilar, 2009; Wood et al., 1976) el aprendizaje de los estudiantes, y de este modo, generar en ellos mayor control y responsabilidad en el proceso. En matemáticas, la profesora busca favorecer el surgimiento de preguntas en los estudiantes, invitándolos abiertamente a consultar (SIAD); mientras que en lenguaje, el docente busca que sus alumnos apliquen los contenidos revisados en el segmento de explicación, a través del desarrollo conjunto de los ejercicios, propiciando de este modo la participación (SIEC).

Es interesante observar que pese a las diferencias encontradas en los segmentos de interactividad de ambas materias, a la base se encuentran configuraciones de actuación similares (EXP/SEG, P-R-F, P-R, P-R-I-C, SEG/PREG-RESP). Lo particular en cada una, al parecer, se relaciona con el abordaje individual o grupal que adopta cada docente: en el caso de matemáticas, focalizado en un solo estudiante, y en el caso de lenguaje centrándose marcadamente en el conjunto de aprendices. Esta característica aparece también en los SI finales de las secuencia didácticas, en donde se otorgan mayores niveles de control y responsabilidad respecto del aprendizaje, a los estudiantes (SIEC, SIEI). En el caso de lenguaje, se realiza mediante el desarrollo grupal de ejercicios de Plan de Redacción, en donde el profesor asume un rol promotor de la actividad conjunta representada en discusiones de todo el grupo; y en el caso de matemáticas, a través del desarrollo individual de ejercicios de ecuaciones, ya sea en el puesto de trabajo, o bien en la pizarra. Lo anterior resultaría coherente con los hallazgos de otras investigaciones que señalan la tendencia a aumentar la aplicación de conocimientos a medida que avanzan en el tiempo los segmentos (Maiz et al., 1996; Onrubia, 1993). Del mismo modo, mostraría la adaptación de las ayudas ofrecidas por los profesores, tal como indican los planteamientos teóricos de Brunner sobre el andamiaje (Brunner, 1991; Guilar, 2009; Wood et al., 1976), y describiría, de acuerdo a la propuesta de Rogoff (1993), a los profesores promoviendo el diálogo y discusión entre los estudiantes, al mismo tiempo que canalizando indirectamente las actividades (Abarca y Astudillo, 2014; Rogoff, 1993). Por otra parte, en ambas materias la trayectoria de las secuencias didácticas se encontró carente de una evolución lógica que contemplara los tres momentos esperados en una clase: introducción, desarrollo y cierre (Rodríguez, 2011). Específicamente, ningún SI hizo referencia al cierre, obviando un momento relevante en el que el docente se encargaría de resumir los contenidos trabajados, identificar los elementos fundamentales, proyectar interrogantes con el fin de orientar hacia nuevos aprendizajes y detectar la comprensión por parte de los estudiantes (Rodríguez, 2011).

La pregunta es una actuación crucial en estas secuencias didácticas, ya sea esta realizada por los estudiantes o por el o la profesora. Está presente en prácticamente todos los tipos de segmentos y su utilización pedagógica es evidente, ya que como actuación no solo colabora en la construcción de prácticamente todos los segmentos de matemáticas y lenguaje, sino que además es la táctica usada por los educadores para transitar hacia un mayor control del aprendizaje por parte del estudiante. Desde esta perspectiva, se podría pensar el uso de la pregunta por parte de los educadores del Preuniversitario Popular PREUMED, no solo en un sentido conservador, como es interpretado de la lectura de Rogoff (1993) que hacen algunos autores (Abarca y Astudillo, 2014) quienes consideran a los patrones de actuación de pregunta del profesor seguida de respuesta del o los estudiantes (P-R) y el monólogo docente (EXP-SEG) como característico del discurso instruccional clásico, y opuesto a la conversación y el diálogo. Las actuaciones y segmentos descritos en 
PREUMED sugieren que estas actuaciones y patrones, si bien pueden encontrarse en prácticamente todos los segmentos, su interpretación en el contexto de la actividad conjunta varía en función de los segmentos de interactividad y de la trayectoria de estos en el tiempo (Coll, 1995, 2002; Coll y Solé, 2002; Coll et al., 1995; Coll et al., 2008; Colomina et al., 2001). En el caso de matemáticas y lenguaje de este preuniversitario, la pregunta permitiría que los estudiantes ejerzan mayor control sobre el contenido del discurso en el aula, es decir desarrollen mayor implicancia en la actividad (Abarca y Astudillo, 2014; Rogoff, 1993) ya que concretamente pueden hablar sobre el tema tratado en la sala de clases, y por ende transformarse también en mediadores respecto de sus pares, como se aprecia en segmentos de ambas materias y se deduce de la teoría.

No es extraño haber encontrado indicadores que se encuentran en coherencia con la propuesta de Rogoff (1993), aun cuando los profesores de este preuniversitario popular no cuentan con formación pedagógica. Al respecto, se observan estrategias que favorecen la participación progresiva de los alumnos, a la vez que colaboran en la preparación del individuo para enfrentarse a escenarios similares. Tal como señala esta investigación y otros autores (Abarca y Astudillo, 2014; Coll, 2002; Rogoff, 1993), no solo en la educación formal operan procesos de andamiaje, sino que en cualquier escenario en los que se desarrollen apoyos en la adquisición de aprendizajes valorados en la sociedad.

Respecto de la problemática de equidad en el acceso, se plantea como hipótesis que la metodología propuesta en este trabajo ayudaría a visibilizar de manera más específica algunos mecanismos psicoinstruccionales a través de los cuales se produce la discriminación masiva de estudiantes para el ingreso a las universidades más selectivas de Chile. Al observar desde una perspectiva psicoinstruccional la evidencia empírica presentada, lo que haría PREUMED sería desarrollar secuencias didácticas tendientes a promover un mayor control de los estudiantes sobre el aprendizaje de contenidos y habilidades exigidas por la PSU. Esto, a través de la mediación que realizan, en tanto educadores y actores culturales, entre el alumno y las herramientas que nuestra cultura ha producido (Wertsch, 1988). De este modo logran alterar precisamente la fórmula a través de la cual en Chile se impide el acceso a la educación superior más prestigiosa: si lo que hacen los preuniversitarios populares es favorecer el control de los estudiantes sobre el aprendizaje necesario para enfrentar la Prueba de Selección Universitaria, es porque evidentemente dichos estudiantes no han tenido la posibilidad de acceder a la cultura representada en el currículum nacional chileno que es evaluado en la PSU, ni tampoco de relacionarse con sujetos mediadores que puedan aproximarlos a dicha producción cultural.

Desde una perspectiva psicoinstruccional, por lo tanto, los problemas de inequidad en el acceso a la educación superior pueden ser enunciados también como inequidad en la producción y distribución (Castro et al., 2014) del acceso a los instrumentos, conceptos, habilidades y conocimientos de la PSU, así como a agentes mediadores que puedan andamiar (Brunner, 1991; Guilar, 2009; Wood et al., 1976) o guiar la participación (Rogoff, 1993) de los estudiantes de sectores populares en su transición desde la enseñanza media hacia la educación superior (González, 2011).

Del mismo modo, la práctica de resistencia a través de la educación (Giroux, 1983) ejercida por PREUMED, puede entenderse desde este análisis como mediación (Vygotsky, 1988), como andamiaje (Brunner, 1991; Guilar, 2009; Wood et al., 1976) y como desenvolvimiento de mecanismos de influencia educativa para favorecer el control del aprendizaje en los estudiantes (Coll, 1995, 2002; Coll et al., 1995; Coll et al., 2008; Coll y 
Solé, 2002; Colomina et al., 2000) y la ampliación de la participación de estos (Onrubia, 2009) en la re-creación de la cultura (Rogoff, 1993).

Finalmente, este mecanismo de influencia educativa y sus indicadores funcionarían como marcas psicoinstruccionales en el mayor o menor control del aprendizaje por parte de los estudiantes, siendo este control un elemento imprescindible en el acceso a la universidad en Chile. En otras palabras, se propone que la exclusión educativa en este país se asociaría no solo a factores económicos, sino que implicaría también una segmentación y aislamiento de una parte de la población estudiantil que no tendría oportunidad de relacionarse con agentes mediadores que colaboren en el desarrollo de proximidad y control sobre estos aprendizajes claves para el ingreso a la enseñanza superior.

Por tanto, lo que estaría haciendo PREUMED sería entrar en la arena política para disputar de facto el ejercicio del control del conocimiento en Chile en favor de los sectores populares. Con ello, no solo estaría colaborando en la construcción de mayor equidad, sino que al mismo tiempo, cuestionando un sistema de selección que pretende detectar a aquellos sujetos con mayores posibilidades de éxito en la enseñanza universitaria (Consejo de Rectores de Universidades Chilenas, 2009). Desde una perspectiva psicoinstruccional, como la desarrollada en este trabajo, lo anterior es entendido más bien como selección de quienes han podido acceder a los mediadores socioculturales que, controlando el aprendizaje valorado en la PSU, son capaces de entregar progresivamente el ejercicio de este control a los y las estudiantes.

\section{REFERENCIAS BIBLIOGRÁFICAS}

Abarca, S. y Astudillo, M. (2014). Desarrollo Cognoscitivo: una lectura intersubjetiva para apropiarnos del conocimiento. Espacio I+D Innovación más Desarrollo, 3(4), 141-159.

Aguilar, A. (2010). Caracterización de las universidades chilenas grupo operativo coordinado por CINDA en relación con la equidad. En Centro Interuniversitario de Desarrollo-CINDA, Grupo Operativo de Universidades Chilenas (Ed.), Diagnóstico y diseño de intervenciones en equidad universitaria (pp. 161 -170). Santiago, Chile. Editorial CINDA.

Bolívar, A. (2005). Equidad educativa y teorías de la justicia. Revista electrónica Iberoamericana sobre calidad, Eficacia y Cambio en Educación, 2(3). 42-69.

Brunner, J. (1991). Actos de significado: más allá de la Revolución cognitiva. Madrid: Alianza.

Brunner, J. (2008). ¿Brecha de resultados o resultado de brechas? Recuperado de http://www. fundacionequitas.org/blog/default.aspx $? \mathrm{id}=34$

Buitrago, O., \& Gutiérrez, M. (2009). Interactividad e Influencia educativa: La construcción de conocimiento en el programa Ondas de Colciencias. Memorias, I congreso Nacional de investigación en educación en ciencias y tecnología, 2009, Junio 22 a 26, Universidad Tecnológica de Pereira.

Cancino, V., \& Donoso, S. (2007). Caracterización socioeconómica de los estudiantes de la educación superior. Revista calidad en la Educación, 26, 203-244.

Castro, M. P., Aranda, C., Castro, C., Lizama, C., Williams, J., \& de Torres, H. (2014). Sistematización de la implementación del Sistema de Ingreso Prioritario de Equidad Educativa (ExCupo de Equidad) en la Carrera de Psicología de la Universidad de Chile 2010-2012. Revista Calidad en la Educación, 40, 338-353.

Catrileo, C. (2010). Red de programas Propedéutico-Unesco. Antecedentes preliminares. Resultados iniciales. Chile: Editorial Universidad Cardenal Silva Henríquez.

Centro Interuniversitario de Desarrollo-CINDA, Grupo Operativo de Universidades Chilenas. (2010). 
Diagnóstico y diseño de intervenciones en equidad universitaria. Chile: Editorial CINDA.

Centro Interuniversitario de Desarrollo-CINDA, Grupo Operativo de Universidades Chilenas. (2011).

Proceso de transición entre la educación media y superior. Chile: Editorial CINDA.

Coll, C. (1995). Elementos para el análisis de la práctica educativa. En C. Genovard, J. Beltrán y F. Rivas (Ed.), Psicología de la instrucción III: Nuevas Perspectivas (pp. 89-102). España: Editorial Síntesis Psicología.

Coll, C. (2002). Lenguaje, actividad y discursos en el aula. En C. Coll, J. Palacios, A. Marchesi (Ed.), Desarrollo Psicológico y Educación (pp.387-414). España: Alianza Editorial.

Coll, C., Colomina, R., Onrubia, J., \& Rochera, M.J. (1992). Actividad conjunto y habla: una aproximación al estudio de los mecanismos de influencia educativa. Revista Infancia y Aprendizaje, 15, 189-232.

Coll, C., Colomina, R., Onrubia, J., \& Rochera, M.J. (1995). Interacción profesor/alumno y adulto/ niño en contextos educativos. En P. Fernández y María de los Ángeles Melero (Eds.), La interacción social en contexto educativos (pp.193-326). España: Siglo Veintiuno.

Coll, C., Onrubia, J., \& Mauri, T. (2008). Ayudar a aprender en contextos educativos: el ejercicio de la influencia educativa y el análisis de la enseñanza. Revista de Educación, 346, 33-70.

Coll, C., \& Solé, I. (2002). Enseñar y aprender en el aula. En Coll, C., Palacios, J. y Marchesi, A. (Eds.), Desarrollo Psicológico y Educación (pp. 357-386). España: Alianza Editorial.

Colomina, R., Mayordomo, R., \& Onrubia, J. (2001). El análisis de la actividad discursiva en la interacción educativa. Algunas opciones teóricas y metodológicas. Revista Infancia y Aprendizaje, 93, 67-80.

Comité Técnico Asesor del Consejo de Rectores de la Universidades Chilenas. (2010). Resultados de la aplicación de pruebas de Selección Universitaria. Admisiones 2006-2010. Santiago, Chile: H. Consejo de Rectores de la Universidades Chilenas.

Consejo de Rectores de las Universidades Chilenas (CRUCH). (2009). Sistema de admisión a las universidades del H. Consejo de rectores de las Universidades Chilenas. Recuperado desde: $\mathrm{http} / / /$ informacioncorporativa.usach.cl/arch/doc/SISTEMAADMISION_PSU2009.pdf

Espinoza, O., \& González, L. (2011). La Educación Superior en Chile. Revista El Pensamiento Universitario, 22, 111-120.

Eyzaguirre, B., \& Le Foulon, C. (2002, junio 9). SIES: un proyecto prematuro. El Mercurio, Cuerpo Artes y letras, pp. E8-E9.

Giroux, H. (1985). Teorías de la Reproducción y la resistencia en la nueva sociología de la educación: un análisis crítico. Cuadernos Políticos, 44, 36-65.

Glatthorn, A. (2006). Curriculum leadership. Development and implementation. California: Sage.

Goldrine, T. \& Rojas, S. (2007). Descripción de la práctica docente a través de la interactividad profesor-alumnos. Revista Estudios Pedagógicos, 2(33), 177-197.

González, E. (2011). La nueva juventud y el proceso de transición entre la educación media y superior. En Centro Interuniversitario de Desarrollo - CINDA, Grupo Operativo de Universidades Chilenas. Proceso de transición entre la educación media y superior (pp. 25-61). Chile: Editorial CINDA.

Guilar, M. (2009). Las ideas de Brunner: "de la revolución cognitiva" a la "revolución cultural". Educere, 44(13), 232-241.

Heredero M., \& Bris, M. (2005). Orientación para la participación. II Encuentro nacional de orientadores. Mérida. Recuperado desde: http://scholar.google.cl/s\&q=m\%C3\%A9rida+II+enc uentro+nacional+de+orientadores $+2005 \& b$ tnG $=$ Buscar \&lr=

Hernández, R., Fernández, C., \& Baptista, P. (2006). Metodología de la Investigación. México: Editorial McGraw-Hill.

Latorre, C., González, L., \& Espinoza, O. (2007). Análisis de la política pública en educación superior desde el punto de vista de la equidad: el caso de Chile. Chile: Fundación Equitas.

Maiz, I., Zarandona, E., \& Arrieta, E. (1996). Mecanismos de influencia educativa y formas de 
MECANISMOS DE INFLUENCIA EDUCATIVA EN UN PREUNIVERSITARIO POPULAR DE SANTIAGO DE CHILE:

CONTROL DEL APRENDIZAJE Y EQUIDAD EN LA EDUCACIÓN SUPERIOR

organización de la actividad conjunta. Revista de Psicodidáctica, 1, 15-25.

Manzi, J. (2006). El acceso segmentado a la educación superior en Chile. En Díaz-Romero (Ed.), Caminos para Educación Superior (pp.187-204). Chile: Fundación Equitas, UNESCO y OREALC.

Martínez, M. (1999). El enfoque sociocultural en el estudio del desarrollo y de la educación. Revista Electrónica de Investigación Educativa, 1(1), 16-37.

Neves de Azevedo, M. (2013). Igualdade e Equidade: qual é a medida da justiça social?, Avaliação, $1(8), 129-150$.

OCDE, Banco Mundial. (2009). La educación Superior en Chile. Revisión de Políticas Nacionales de Educación. Autor.

Onrubia, J. (1993). Interactividad e influencia educativa en la enseñanza/aprendizaje de un procesador de textos: una aproximación teórica y empírica. Anuario de Psicología , 58, 83-103.

Onrubia, J. (2009). Transformar para adaptar, adaptar para incluir: una mirada psicoeducativa a la educación inclusiva. En C. Giné. (Ed.), Una educación Inclusiva: de la exclusión a la plena participación de todo el alumnado (pp. 49-62). España: Editores Universitat de Barcelona, Institut de Ciències de l'Educació, ICE, Horsori.

Rodríguez, M. (2011). Metodologías de enseñanza en las EEES: de la enseñanza magistral al protafolio. Revista Tendencias Pedagógicas, 17, 83-103.

Rogoff, B. (1993). Aprendices del pensamiento: el desarrollo cognitivo en el contexto social. España: Paidós.

Rosemberg, C. R. (2000). El aprendizaje lingüístico y discursivo: la incidencia de la interacción social. Revista Internacional Paideia, 4, 85-94.

Vygostky, L (1988). El desarrollo de los procesos psicológicos superiores. México: Grijalbo.

Wertsch, J. (1988). Vygostky y la formación social de la mente. Buenos Aires: Ediciones Paidós.

Williamson, C., \& Rodríguez, C. (2010). Equidad, inequidad y educación superior: aprendizaje de un preuniversitario para jóvenes de la educación de adultos. Estudios Pedagógicos, 36(2), 251-267.

Wood, D., Brunner, J.S., \& Ross, G. (1976). The role of tutring im problem solving. Journal of Child Psychology and Psychiatry, 7, 89-100.

Zarandona, E., Arrieta, E., \& Maiz, I. (1997). Mecanismos de influencia educativa en los intercambios diádicos -profesora/alumno/a- en un aula de preescolar. Revista de Psicodidáctica, 3, 83-91. 